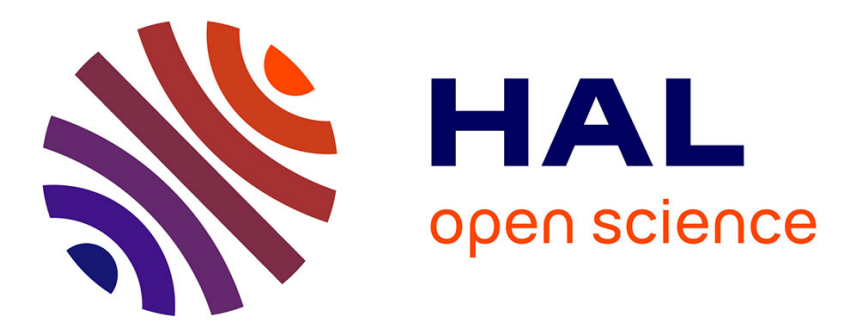

\title{
Spatial structuring and size selection as collective behaviours in an agent-based model for barchan fields.
}

Mathieu Génois, Pascal Hersen, Sylvain Courrech Du Pont, Guillaume

Grégoire

\section{- To cite this version:}

Mathieu Génois, Pascal Hersen, Sylvain Courrech Du Pont, Guillaume Grégoire. Spatial structuring and size selection as collective behaviours in an agent-based model for barchan fields.. The European Physical Journal B: Condensed Matter and Complex Systems, 2013, 86 (11), pp.447. 10.1140/epjb/e2013-40710-2 . hal-00869412

\section{HAL Id: hal-00869412 https://hal.science/hal-00869412}

Submitted on 3 Oct 2013

HAL is a multi-disciplinary open access archive for the deposit and dissemination of scientific research documents, whether they are published or not. The documents may come from teaching and research institutions in France or abroad, or from public or private research centers.
L'archive ouverte pluridisciplinaire HAL, est destinée au dépôt et à la diffusion de documents scientifiques de niveau recherche, publiés ou non, émanant des établissements d'enseignement et de recherche français ou étrangers, des laboratoires publics ou privés. 


\title{
Spatial structuring and size selection as collective behaviours in an agent-based model for barchan fields.
}

\author{
Mathieu Génois ${ }^{12 a}$, Pascal Hersen ${ }^{1}$, Sylvain Courrech du Pont ${ }^{1}$, and Guillaume Grégoire ${ }^{12 b}$ \\ 1 Laboratoire Matière et Systèmes Complexes (MSC),Univ. Paris-Diderot, CNRS UMR 7057, 75205 Paris CEDEX 13 \\ 2 Academy of Bradylogists
}

September 18, 2013

\begin{abstract}
In order to test parameters of the peculiar dynamics occurring in barchan fields, and compute statistical analysis over large numbers of dunes, we build and study an agent-based model, which includes the well-known physics of an isolated barchan, and observations of interactions between dunes. We showed in a previous study that such a model, where barchans interact through short-range sand recapture and collisions, reproduces the peculiar behaviours of real fields, namely its spatial structuring along the wind direction, and the size selection by the local density. In this paper we focus on the mechanisms that drives these features. In particular, we show that eolian remote sand transfer between dunes ensures that a dense field structures itself into a very heterogeneous pattern, which alternates dense and diluted stripes in the wind direction. In these very dense clusters of dunes, the accumulation of collisions leads to the local emergence of a new size for the dunes.
\end{abstract}

\section{Introduction}

Barchans are very common dunes [1], found not only in Earth deserts and at the bottom of the ocean, but also on other bodies in the solar system like Mars [2], Venus and Titan [3]. Physics of an isolated barchan is now quite well understood [4]. Barchans form when a small amount of sand is blown by an unidirectional flow on a flat, nonerodible surface. Their specific crescentic shape (Fig. 1) is due to the presence of a recirculation bubble at the downwind side, which acts as a sand trap, whereas sand is blown from the dune at its horns. Barchans can also catch sand transported by the wind, but though a fixed point exists for their size, they are unstable structures. They can either shrink and disappear, if they are smaller than the equilibrium size and thus catch less sand than they lose, or grow to become giant barchans if they are bigger than the equilibrium size and thus catch more sand than they lose [4].

But barchans are almost never isolated. They are found in fields that sometimes gather thousands of dunes (Fig. 1), which sizes follow what seems to be a log-normal distribution [5], very different from what would be an assembly of giant barchans. Furthermore, some barchan fields - for example in Morocco (Fig. 1) - exhibit odd features, that cannot be explained by the simple juxtaposition of single dune dynamics. They are structured in sharp, well-defined corridors along the wind direction, each characterised by a specific density and mean size for the dunes. These two

\footnotetext{
${ }^{a}$ genois.mathieu@gmail.com

${ }^{b}$ guillaume.gregoire@univ-paris-diderot.fr
}
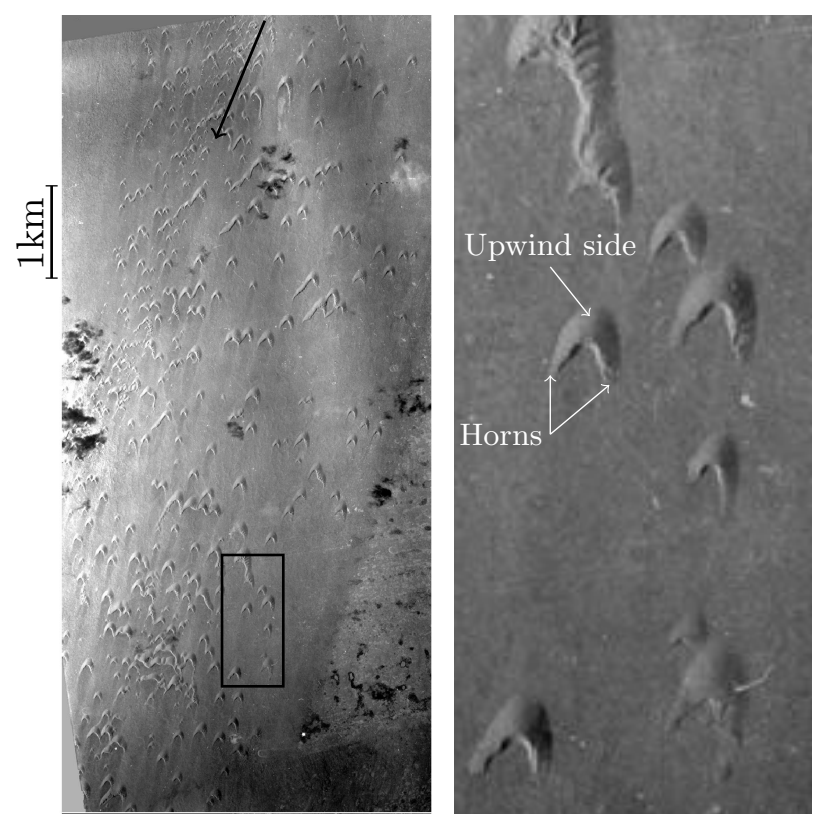

Fig. 1. Barchan field in Western Morocco. The black arrow indicates the wind direction. The right part is a zoom of the black rectangle area.

quantities seem to be correlated: the denser the corridor, the smaller the mean size [6]. External parameters - wind, sand and soil properties - cannot be held responsible, as it has been shown that they usually are constant all over the field $[6]$. 
In a field, barchans interact with each other through highly non linear events, that perturb their isolated behaviour by redistributing the sand and sometimes annihilating or creating dunes. These interactions may therefore be responsible for the peculiar features of the field. This hypothesis has been made previously [7] and used in several models of multiple types - continuous [8-11,7], cellular automatons [12,13], agent-based models [14]. In a previous paper [15] we built a model of the last kind, where particles are the dunes themselves. We were then able to simulate large assemblies and compute and study long time scales, global behaviours for the field. The model takes into account the physics of isolated barchans and computes remote interactions between dunes through sand recapture and collisions. We have shown that the implementation of these interactions is sufficient for the system to reproduce both the spatial structuring of the field along the wind direction, and the local anticorrelation between the density and the mean size of dunes.

In this paper, we seek to understand by which mechanisms these features emerge. We will describe the model in details, in particular the peculiar ways two dunes can interact. The model is controlled by eight physical factors, from which we build six independent adimensional parameters. The effects of these parameters are precisely described, especially for the two that control the phenomenology of collisions. We then recapitulate the two asymptotic behaviours - dilute and dense - for the field, previously described in [15], and relate their characteristics to the microscopic dynamics of the dunes. We show that these features have an impact on the behaviour of large scale quantities, and we take the example of the total volume of sand in the system, which evolution signs very accurately the cross-over between dilute and dense dynamics. We then focus in the dense regime on the spatial structuring of the field into dense stripes along the wind direction, due to the remote interaction between dunes, and the size selection in these structures, which is the result of the collision processes, amplified by the spatial structuring. We finally show that such size selection can emerge from the simple, random accumulation of local interaction, and thus be considered as a collective phenomenon.

\section{Model of a barchan field}

The model is agent-based. The barchan field is seen as an assembly of agents - dunes - which interact with each other through sand capture and collisions. The whole phenomenology of the model is based on observations from real fields $[6,4]$ and from small-scale experiments under water $[16,17]$. We build a minimal model, i.e. we include only the essential ingredients for the system dynamics, with a limited number of parameters. The model is therefore built with strong assumptions. The point of our work is to show that a simple model can reproduce the global, non trivial behaviours of this system. We seek to understand the underlying mechanisms of these behaviours, using a minimum set of parameters.
Barchans can be considered as self-similar objects, so that their geometry is fully described by only one of their dimensions. In our case we choose their width $w$. Each agent is thus a cube, characterised by its position $(x, y)$ and its width $w$ at each time $t$. This is a strong approximation of the physics of one barchan, as we do not consider its particular geometry, aside from the specific behaviours it generates. The cubic agents have a volume $w^{3}$ and follow the physics of isolated barchans. The coordinates $x$ and $y$ and the width $w$ are continuous variables, whereas time is discretised in time steps of value $\Delta t$.

The wind direction sets the $y$ axis of the field. The wind blows in the direction of decreasing $y$. We consider the field far away from its source, so that there is no global sand flux on the field. The volume of an isolated dune evolves only because of sand loss, according to:

$$
w^{3}(t+\Delta t)=w^{3}(t)-\Phi \Delta t
$$

where the constant $\Phi$ is the volume of sand each dune loses, per unit of time. For a real barchan, sand loss occurs only at its two horns, in two cones due to diffusion processes. In the model, we do not take into account the spatial repartition of this loss, and consider that the sand loss is uniform along the width of the dune.

The law (1) is valid until the dune reaches a minimum size $w_{m}$. Under this size - which existence is verified for real dunes [16] - a barchan can no longer keep its particular shape and becomes a simple dome that quickly loses sand and vanishes [4]. Thus, in the model, whenever a dune becomes smaller than $w_{m}$, it is removed from the field.

Each dune moves along the wind direction with a speed determined by:

$$
v=\frac{\alpha}{w}
$$

where $\alpha$ is a mobility coefficient for the dunes, related to characteristics of the wind and the sand grains. This inverse law is typical for barchan dunes [4].

A real barchan field is structured along the wind direction, from its source, where dunes are injected, to its vanishing point. With the model, we seek to study potential steady states for the field dynamics, so we impose periodical conditions on both directions, and lose the structure of a real field. However, we still need a source term in order to feed the field, so we add a dune injection term. Dunes with a fixed size $w_{n}$ are nucleated in the field, at random time and position, at a mean rate $\lambda$ by surface unit. This strong, random, external forcing also ensures that every behaviour of the system will be robust.

Interactions exist between dunes through sand capture. The sand lost by an upwind dune can be caught by a downwind one, if the sand has not yet be blown away by lateral diffusion. We thus define a maximum distance of interaction $d_{0}$, which allows us to tune the range of this interaction. Whenever two dunes overlay along the direction perpendicular to the wind, and the distance between them along the wind direction is less than $d_{0}$ (see Fig 2a), the downwind one catches a fraction of the sand lost by 


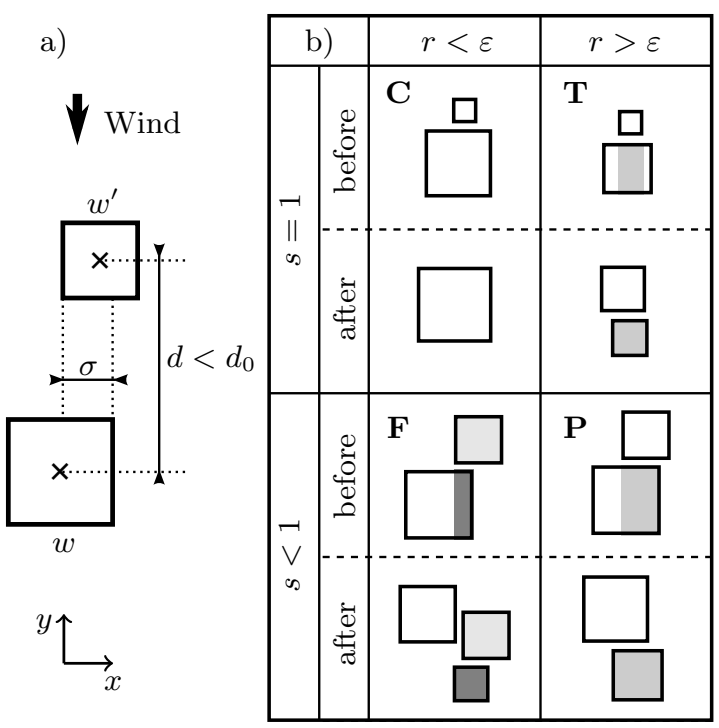

Fig. 2. Diagram of dunes interactions. a) Sand capture. b) Collisions : C: coalescence, $\mathbf{F}$ : fragmentation, $\mathbf{T}$ : sand redistribution with total overlap, $\mathbf{P}$ : sand redistribution with partial overlap. For each type is drawn the situation before and after the collision. The levels of grey indicate the sections of the dunes which merge during the collision.

the upwind one, proportional to the ratio of the overlay length $\sigma$ and the size of the upwind dune:

$$
Q_{\Phi}=\frac{\sigma}{w^{\prime}} \Phi
$$

The change of size of the dune is immediate, in order to conserve its aspect ratio.

In addition, dunes can collide. Indeed, due to the particular law for their speed (eq. (2)), small dunes travel faster than large ones. In the model, a collision occurs when two dunes overlay along the $x$ direction, and the centre of mass of the upwind one runs over the downwind one's. As dunes in the model do not have hard cores and are merely points with one variable that mimics their spatial extent, they can overlap. So a collision event consists of three parts: an overlap time before the collision, the colliding event itself, and an overlap time after the collision, until the dunes engendered by the collision go apart or collide again. This way of simulating collisions between dunes is in the end a good way to also simulate the lifetime of the complex structures that are formed in real fields whenever two dunes collide [18].

The phenomenology of real collisions is very wide. We summarise it into three elementary types of collision: coalescence, sand redistribution, and fragmentation. These basic types can combine to solve complex collisions involving more than two dunes and short-time series of collisions. Collision types are derived from the main mechanism of a collision: the presence of the upwind dune modifies the wind flow, which destabilises the section of the downwind dune it overlays, leading to its ejection. The remaining part may then be gathered by the upwind dune to form a new one. As we restrict the collision process to this only mechanism, the total volume of sand is conserved during a collision, and the collision phenomenology is entirely determined by the local geometry, i.e. the relative positions and sizes of the two dunes. Two parameters $r$ and $s$ characterise this geometry (see Fig. 2a) by comparing the overlay length $\sigma$ to the sizes of the dunes that collide:

$$
\begin{aligned}
& r=\frac{\sigma}{w} \\
& s=\frac{\sigma}{w^{\prime}}
\end{aligned}
$$

If the overlay is complete $(s=1)$, there are two possibilities. If the upwind dune is big enough compared to the other one, i.e. $r$ greater than a certain limit value $\varepsilon_{t}$, the collision occurs exactly as previously described: the flow destabilises the section of the downwind dune, which is ejected, while the remaining parts and the upwind dune merge into a new one. This is the sand redistribution with full overlap (case $\mathbf{T}$ in Fig. 2). As the number of dunes is conserved, this collision only consists in a simple redistribution of the sand. If the upwind dune is too small compared to the other one $\left(r<\varepsilon_{t}\right)$, the flow is not able to destabilise the downwind one, and the two simply merge. That is the coalescence (case $\mathbf{C}$ in Fig. 2).

When the overlay is not complete $(s<1)$, there are also two possibilities. If the collision is grazing, i.e. $r$ is smaller than a certain limit value $\varepsilon_{p}$, the destabilisation process is the same, but the two remaining dunes stay separated. It is the fragmentation (case $\mathbf{F}$ in Fig. 2). If the collision is not grazing $\left(r>\varepsilon_{p}\right)$, the phenomenology is the same as when $s=1, r>\varepsilon_{t}$ : the section of the downwind dune that is overlapped is ejected, and the remaining part and the upwind dune merge. It is the sand redistribution with partial overlap (case $\mathbf{P}$ in Fig. 2).

The diagram in Fig. 2b summarised the phenomenology of collisions. Each type of collision conserves the total volume of sand during the event. The new volume of each dune is simply the sum of the section volumes that compose it. The positions of the dunes after a collision are also computed according to the configuration of the collision. As dunes are built by one or several sections of the colliding dunes, their lateral position is simply the barycenter of the sections they are built from. Their longitudinal coordinate is set to the one of the former downwind dune. Finally, the algorithm combines these three types of collision to solve more complex events, as multiple dunes collisions or successive collisions between dunes coming from the same initial pair. Modifications of the volumes described in Fig. 2b can be summarised by the following graph (each pair of brackets stands for a single dune): 


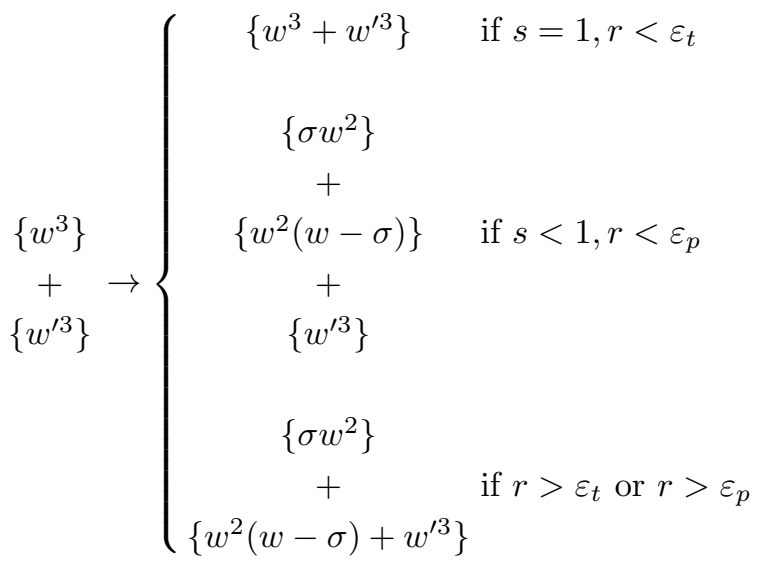

The evolution of the volume of each dune can therefore be expressed by a single equation:

$$
w_{k}^{3}(t+\Delta t)=w_{k}^{3}(t)+\left(-1+\sum_{j} \frac{\sigma_{k j}}{w_{j}}\right) \Phi \Delta t+\mathbf{C}
$$

where the sum is done on all dunes $j$ that supply the dune $k$, and $\mathbf{C}$ stands for the effect of all collisions the dune $k$ undergoes.

\section{Parameters of the model}

Eight physical parameters control the dynamics of the model. They are:

$-w_{n}[L]:$ size of nucleated dunes,

- $w_{m}[L]:$ minimum size of dunes,

$-d_{0}[L]$ : limit distance of interaction,

$-\Phi\left[L^{3} T^{-1}\right]$ : volume of sand lost by each dune at each time step,

$-\lambda\left[L^{-2} T^{-1}\right]$ : rate of dune nucleation, by surface unit,

$-\alpha\left[L^{2} T^{-1}\right]$ : mobility coefficient for barchans,

$-\varepsilon_{t}$ (no dim.): limit parameter for collisions when $s=1$.

$-\varepsilon_{p}$ (no dim.): limit parameter for collisions when $s<1$.

The first three are typical lengths, the next three define three time scales for the dynamics. As there are only two dimensions in the system - length and time - and $\varepsilon_{t}$ and $\varepsilon_{p}$ are already dimensionless, we build four dimensionless control parameters: two aspect ratios and two time ratios.

\subsection{Aspect ratios}

With the three typical lengths in the model, we define two aspect ratios:

$$
\begin{aligned}
& \delta=\frac{w_{m}}{w_{n}} \\
& \Delta=\frac{w_{n}}{d_{0}}
\end{aligned}
$$

$\delta$ compares the minimum size of dunes to the nucleation size. It sets the range of dune sizes. $\Delta$ compares the nucleation size to the limit distance of eolian interaction. It controls the range of this interaction.

\subsection{Time ratios}

Three typical times for the dynamics of the field can be built. We define the time for a dune of size $w_{n}$ to disappear by sand loss only:

$$
\tau_{d}=\frac{w_{n}^{3}-w_{m}^{3}}{\Phi},
$$

the mean time of dune nucleation on a surface $d_{0}^{2}$ :

$$
\tau_{n}=\frac{1}{\lambda d_{0}^{2}},
$$

and a collision time, defined as the time for a dune of size $w_{m}$ to catch a dune of size $w_{n}$ within a distance $d_{0}$, assuming that there is no sand loss:

$$
\tau_{c}=\frac{d_{0}}{\alpha}\left(w_{m}^{-1}-w_{n}^{-1}\right)^{-1} .
$$

The two remaining control parameters compare these times:

$$
\xi=\frac{\tau_{d}}{\tau_{n}}=\frac{w_{n}^{3}-w_{m}^{3}}{\Phi} \lambda d_{0}^{2}
$$

and:

$$
\eta=\frac{\tau_{d}}{\tau_{c}}=\frac{w_{n}^{3}-w_{m}^{3}}{\Phi} \frac{\alpha}{d_{0}}\left(w_{m}^{-1}-w_{n}^{-1}\right)
$$

These parameters control the dynamics of the field. $\xi$ compares the sand loss to the dune injection, and so the relative importance of the dissipation and the forcing. $\eta$ compares the relative importance of isolated dynamics sand loss - to collision dynamics.

\subsection{Limit parameters for the collisions}

\subsubsection{Diagram of the collisions}

The values of $\varepsilon_{t}$ and $\varepsilon_{p}$ determinate the relative proportion of each type of collision. These phenomena are entirely deterministic, and are based on the geometry of the collision, namely the relative offset and sizes of the dunes. We thus calculate the diagram of the collisions, depending on the size ratio of the dunes $\pi_{i}$ and their lateral offset $\theta_{i}$ before the collision, defined as:

$$
\begin{gathered}
\pi_{i}=\frac{w_{i}^{\prime}}{w_{i}} \\
\theta_{i}=\frac{\left|x_{i}-x_{i}^{\prime}\right|}{w_{i}}
\end{gathered}
$$

where the prime marks the variables of the upwind dune, and the subscript $i$ designates values before the collision.

The diagram is shown on Fig. 3 . The line $\mathbf{d}_{\mathbf{1}}$ of equation:

$$
\pi_{i}=2 \theta_{i}-1
$$

delimits the domain of the existence for the collision, given by $\left|x_{i}-x_{i}^{\prime}\right|<\left(w_{i}+w_{i}^{\prime}\right) / 2$. Beyond this limit, the upwind 
dune no longer overlaps the downwind one, and there is no collision. In the same way, there is also the limit $\pi_{i}<1$, which ensures that the upwind dune is the smallest and therefore the fastest. Finally, both parameters are strictly positive by definition, so $\pi_{i}>0$ and $\theta_{i}>0$,

The line $\mathbf{d}_{\mathbf{2}}$ of equation:

$$
\pi_{i}=-2 \theta_{i}+1
$$

marks out the full overlap (on the left), and partial overlap (on the right) areas. Its equation is derived from the limit on the lateral offset $\left|x_{i}-x_{i}^{\prime}\right|<\left(w_{i}-w_{i}^{\prime}\right) / 2$.

Into the full overlap area, the limit $r=\varepsilon_{t}$ between coalescence and sand redistribution can be rewritten as $\sigma_{i}=\varepsilon_{t} w_{i}$. As $s=1, \sigma_{i}=w_{i}^{\prime}$ and the two areas are marked out in the diagram by the line $\mathbf{d}_{\mathbf{3}}$ of equation:

$$
\pi_{i}=\varepsilon_{t}
$$

Into the partial overlap area, the limit $r=\varepsilon_{p}$ between fragmentation and sand redistribution becomes $\sigma_{i}=\varepsilon_{p} w_{i}$. As $s<1, \sigma_{i}$ depends on the offset $\left|x_{i}-x_{i}^{\prime}\right|$, and the equation of the line $\mathbf{d}_{\mathbf{4}}$ that delimits these behaviours is given by:

$$
\pi_{i}=2\left(\theta_{i}+\varepsilon_{p}\right)-1
$$

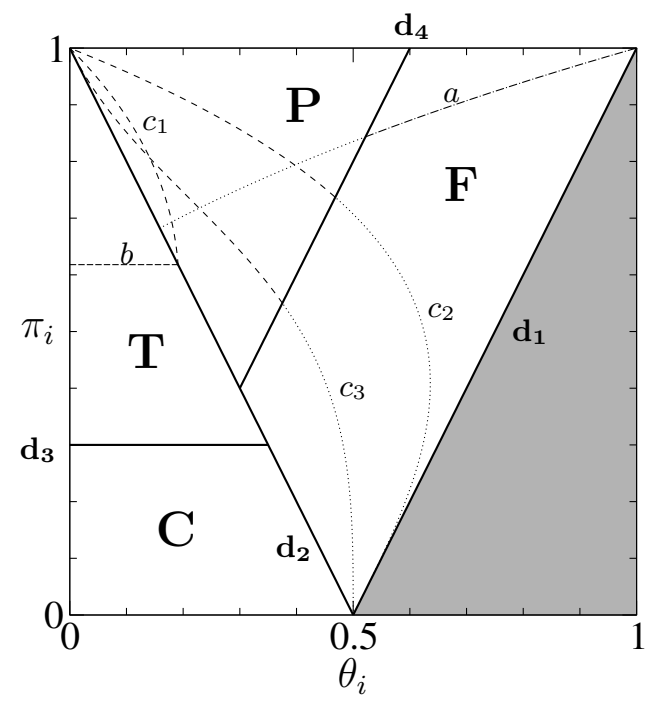

Fig. 3. Diagram of the collisions, depending on the intial offset ratio $\theta_{i}$ and size ratio $\pi_{i}$, for $\varepsilon_{t}=0.3$ and $\varepsilon_{p}=0.4$. As $\mathbf{d}_{4}$ is mobile, the curves $a, c_{2}$ and $c_{3}$ are drawn to their full extent. The unused parts for this particular example are plot in dots.

These four lines define the three types of collision: coalescence $(\mathbf{C})$, fragmentation $(\mathbf{F})$, and sand redistribution with a distinction between redistribution with total $(\mathbf{T})$ and partial $(\mathbf{P})$ overlap. Among these limits, only $\mathbf{d}_{\mathbf{3}}$ and $\mathbf{d}_{4}$ depend on the limit parameters $\varepsilon_{t}$ and $\varepsilon_{p}$, and are thus mobile. We can then calculate, within each pair of collisions, the particular values of $\varepsilon_{t}$ and $\varepsilon_{p}$ for which the surface of the regions in the diagram, and so the probabilities of the collisions, are equal. This is achieved when:

$$
\varepsilon_{t}=\varepsilon_{p}=1-\frac{1}{\sqrt{2}} \simeq 0.293
$$

\subsubsection{Sand redistribution \& Dune shifts in collisions}

We look at how collisions affect the relative offsets in size and position between two dunes. This will give us information about how collisions modify both the size and the spatial distributions of dunes in the field. We define the relative difference $\omega$ between the sizes as:

$$
\omega=\frac{w-w^{\prime}}{w}=1-\pi
$$

where $w$ and $w^{\prime}$ respectively designate the downwind and the upwind dune before the collision. We calculate the different limits $\omega_{f}>\omega_{i}$ (the difference between the sizes of the dunes grows with the collision), $\omega_{f}=0$ (after the collision the two dunes have the same size, meaning there will probably be another collision), and $\theta_{f}>\theta_{i}$ (the dunes are more apart after the collision), using the deterministic phenomenology of the collision for the volumes (eq. (6)) and the reconstruction of the centre of mass through the geometry of the collision.

For the coalescence $(\mathbf{C})$, since there is only one dune left after the collision, we have by definition $\pi_{f}=\theta_{f}=0$, and thus no particular limit in the behaviour.

For the fragmentation $(\mathbf{F})$, we do not consider the small dune ejected. We define the ratios with the upwind dune and the remaining part of the downwind dune. Thus, we always have $\omega_{f}<\omega_{i}$, meaning that difference between the sizes always decreases, and $\theta_{f}>\theta_{i}$, meaning that the offset distance between the dunes increases. Yet $\omega_{f}$ can be positive or negative. We uncover two sub-regions of this area, separated by the curve $a$ of equation:

$$
\theta_{i}=\pi_{i}^{3}+\frac{\pi_{i}-1}{2}
$$

Around this limit, dunes have roughly the same size after the collision, so they probably will collide again.

For sand redistribution, arbitrary rules are necessary to define which dune is which. The ejected part of the former downwind dune will be considered as the new upwind dune of size $w^{\prime}$, while the fusion of the upwind dune and the remaining parts of the downwind one will be considered as the new downwind dune of size $w$.

When the overlap is total $(\mathbf{T})$, we always have $\omega_{f}<\omega_{i}$ and $\theta_{f}<\theta_{i}$. Both the difference between the sizes and the distance between the dunes decrease during the collision. As for the fragmentation, $\omega_{f}$ can be positive or negative. The curve $b$ of equation:

$$
\pi_{i}=\pi_{0}=\frac{\sqrt{5}-1}{2} \simeq 0.618
$$

delimits the sub-region where $\omega_{f}>0$ (below) and the one where $\omega_{f}<0$ (above). This limit also ensures the 
existence of a particular value for $\varepsilon_{t}$, as $\mathbf{d}_{\mathbf{3}}$ is mobile. If $\varepsilon_{t}>\pi_{0}$, the behaviour set by $\omega_{f}>0$ disappears.

Partial overlap sand redistribution $(\mathbf{P})$ is more complicated, as all behaviours are possible. There are therefore three limits. The line $c_{1}$ of equation:

$$
\theta_{i}=\frac{\pi_{i}-\pi_{i}^{3}}{2}
$$

marks out the $\omega_{f}>0$ (right part) and $\omega_{f}<0$ (left part) areas. The line $c_{2}$ of equation:

$$
\theta_{i}=\frac{1+\pi_{i}-\pi_{i}^{3}+\pi_{i}^{4}-2 \pi_{i}^{6}}{2\left(1+\pi_{i}^{3}\right)}
$$

marks out the $\omega_{f}>\omega_{i}$ (right part) and $\omega_{f}<\omega_{i}$ (left part) areas. The line $c_{3}$ of equation:

$$
\theta_{i}=\frac{-3 \pi_{i}^{3}+\pi_{i}+\sqrt{9 \pi_{i}^{6}-10 \pi_{i}^{4}+4 \pi_{i}^{3}+\pi_{i}^{2}-4 \pi_{i}+4}}{4}
$$

marks out the $\theta_{f}>\theta_{i}$ (left part) and $\theta_{f}<\theta_{i}$ (right part) areas.

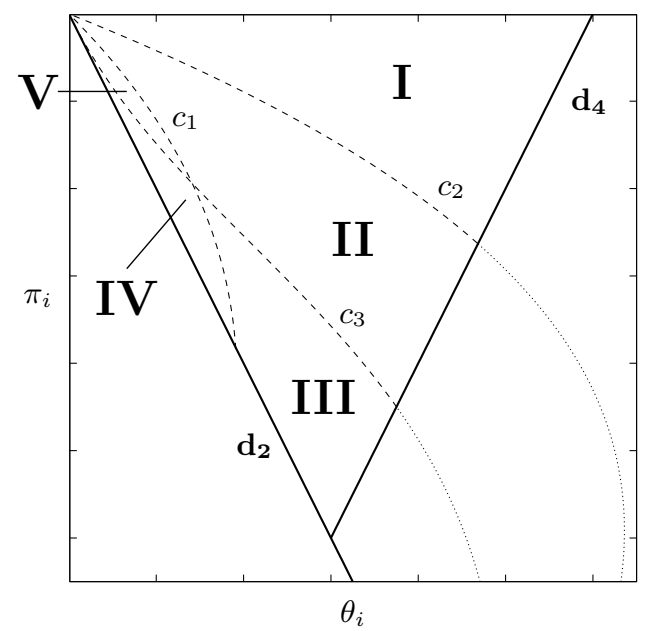

Fig. 4. Detail of the sand redistribution with partial overlap collision area $(\mathbf{P})$ of the diagram of collisions.

\begin{tabular}{|c|c|c|c|}
\hline I & $\omega_{f}>0$ & $\omega_{f}>\omega_{i}$ & $\theta_{f}<\theta_{i}$ \\
\hline II & $\omega_{f}>0$ & $\omega_{f}<\omega_{i}$ & $\theta_{f}<\theta_{i}$ \\
\hline III & $\omega_{f}>0$ & $\omega_{f}<\omega_{i}$ & $\theta_{f}>\theta_{i}$ \\
\hline IV & $\omega_{f}<0$ & $\omega_{f}<\omega_{i}$ & $\theta_{f}>\theta_{i}$ \\
\hline $\mathbf{V}$ & $\omega_{f}<0$ & $\omega_{f}<\omega_{i}$ & $\theta_{f}<\theta_{i}$ \\
\hline
\end{tabular}

Table 1. The five different behaviours of the $\mathbf{P}$ collision.

These limits define five different combinations as shown on Figure 4. Table 1 recapitulates these behaviours. In regions IV and $\mathbf{V}$ we have $\omega_{f}<0$. The difference in sizes increases only in region $\mathbf{I}$, in all the other regions this gap decreases. Finally, the collision makes dunes move away from each other in regions III and IV, while it brings them closer in regions $\mathbf{I}, \mathbf{I I}$ and $\mathbf{V}$.

The geometry of these limits also ensures that there exists a particular value for $\varepsilon_{p}$. If $\varepsilon_{p}>0.806$, the $\mathbf{d}_{\mathbf{4}}$ limit goes beyond the intersection of $\mathbf{c}_{\mathbf{1}}$ and $\mathbf{c}_{\boldsymbol{3}}$ and the region III disappears.

\subsection{Settings of the model}

In this study, we focus on the evolution of the dynamics when $\Phi$ changes, i.e. when the lifetime of the dunes changes. Both $\xi$ and $\eta$ are inversely proportional to $\Phi$, so $\eta \propto \xi$ and we choose $\xi$ to follow the evolution of the system. The two aspect ratios $\delta$ and $\Delta$ are kept constant, as the limit overlay values $\varepsilon_{t}$ and $\varepsilon_{p}$. These parameters are purely geometric, their effect on the behaviour should only modulate the underlying dynamics set by $\xi$ and $\eta$, modulations we are not interested in for this study.

The field is a square of size $32 \times 32$. The length scale is $d_{0}$, which value is arbitrary fixed to 1 . We then fix $\Delta=0.1$ to ensure that interactions between dunes are of moderate range, and $\delta=0.1$ to mimic the observed range of sizes for barchans. For the simulations to stay relevant, we impose that the fastest dune in the field takes 10 time steps to travel along a length unit $d_{0}$. This fixes $\alpha$ to 0.001 , as the value of one time step $\Delta t$ is set to 1 . The nucleation is rather low, one dune every two time steps for the whole field, which sets the value for $\lambda$. Finally, we impose $\varepsilon_{t}=$ $\varepsilon_{p}=0.5$ to ensure all described collision behaviour exist. Parameters values are summarised in Table 2.

\begin{tabular}{|c|c|}
\hline$d_{0}$ & 1 \\
\hline$w_{n}$ & 0.1 \\
\hline$w_{m}$ & 0.01 \\
\hline$\Delta t$ & 1 \\
\hline$\alpha$ & $10^{-3}$ \\
\hline$\lambda$ & $2^{-11}$ \\
\hline$\Phi$ & {$\left[7 \times 10^{-8} ; 5 \times 10^{-5}\right]$} \\
\hline$\varepsilon_{t}$ & 0.5 \\
\hline$\varepsilon_{p}$ & 0.5 \\
\hline \hline$\delta$ & 0.1 \\
\hline$\Delta$ & 0.1 \\
\hline$\xi$ & {$\left[10^{-2} ; 7\right]$} \\
\hline$\eta$ & {$[1.8 ; 1260]$} \\
\hline
\end{tabular}

Table 2. Parameters of the simulations. Based on typical sizes and speeds of dunes in Morocco, a unit of length corresponds to $\sim 1 \mathrm{~km}$ and a unit of time corresponds to $\sim 1$ year.

Simulations are usually carried for $10^{5} \Delta t$. In the following results, means are of two sorts: field scale quantities 
are averaged both in time and over several simulations; local quantities are averaged on the field, in time and over several simulations.

\section{Dilute dynamics}

For each $\Phi$, the system reaches a fluctuating, out of equilibrium, steady state. This indicates that local dissipation always compensates the large scale forcing. The system adjusts its dynamics by itself.

These steady states are of two kinds [15]. In the dilute regime $(\xi \ll 1)$, dunes live not long enough to interact with their neighbours, which are too far away. The simple balance between $\Phi$ and $\lambda$ leads to the stationary state. As dunes do not interact, the whole dynamics can be analytically derived from this balance [15]. In particular, the mean density of the field is then:

$$
\rho=\frac{\xi}{d_{0}^{2}}=\xi^{*}
$$

The mean density grows linearly with $\xi$, and is in fact exactly the typical density $\xi^{*}$ defined by the comparison between the time scales of sand loss and nucleation. Simulations concur with this results. Providing that the diluted field hypothesis is valid, i.e. there are no collisions (Fig. 5a), the density grows linearly with $\xi$.

From eq. 1 and the fact that the nucleation rate $\lambda$ is a constant, we can derive the dune size distribution, which is given by:

$$
p(w)=\frac{3 w^{2}}{w_{n}^{3}-w_{m}^{3}}
$$

As seen on Fig. 5b, this distribution is quadratic, and thus tends to promote large dunes. Dunes are nucleated at a size $w_{n}$, and then simply lose sand, which generates the smooth decrease of the distribution, until dunes reach the minimum size $w_{m}$.
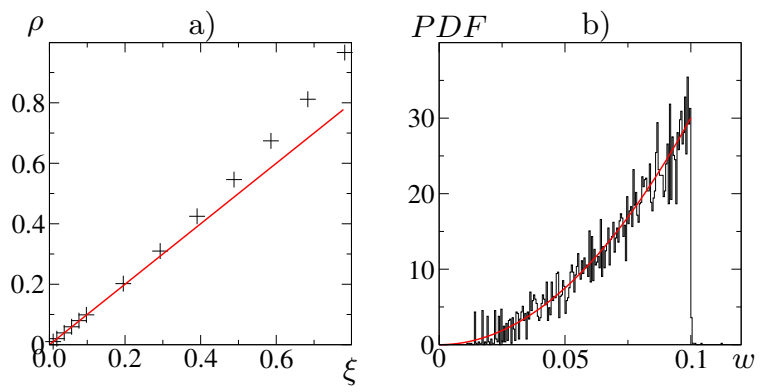

Fig. 5. a) Mean density of the field as a function of $\xi$. The red line shows $\rho=\xi^{*}$. b) Size distribution for $\xi=0.488$, (see Table 2 for the other parameters). The red line shows the theoretical distribution.

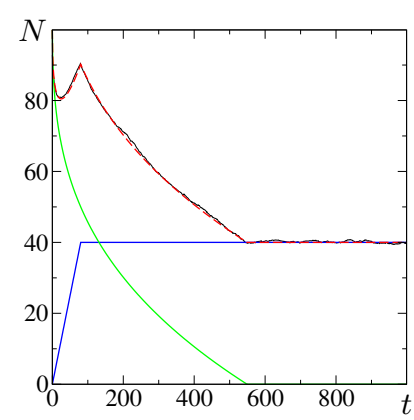

Fig. 6. Transient behaviour for $\xi=0.039$ and $N_{0}=120$ (see Table 2 for the other parameters). Comparison between the simulation (-) and the theory (-), calculated as the sum of the evolution of the initial population $(-)$ and the nucleated population (-).

When the non-interacting dunes hypothesis is verified at each moment, one can analytically derive the whole evolution of the system, including the transient behaviour. The main idea of this calculus is that the whole population can be divided into two sub-populations which - by definition of the dilute hypothesis - do not interact with each other:

- the initial population, which decreases and vanishes at a finite time $t_{i}$,

- the nucleated population, which does not exist at the initial moment $t_{0}$, and replaces entirely the initial population at $t_{i}$.

The time of disappearance $t_{i}$ is equal to the time at which the biggest dune of the initial population disappears. This time is given by:

$$
t_{i}=\frac{\left(2 w_{n}-w_{m}\right)^{3}-w_{m}^{3}}{\Phi}
$$

since the sizes of the initial population follow a uniform distribution centred on $w_{n}$ with a cutoff at $w_{m}$. By deriving the evolution of the size distribution for each population, one finds that the number of dunes of the initial population decreases according to:

$$
N_{i}(t)=\frac{N_{0}}{2\left(w_{n}-w_{m}\right)}\left(2 w_{n}-w_{m}-\left(w_{m}^{3}+\Phi t\right)^{1 / 3}\right)
$$

where $N_{0}$ is the initial number of dunes in the field, until $N_{i}$ reaches 0 at $t_{i}$. In the same time, the nucleated population appears, following (with $S=L \ell$ the surface of the field):

$$
N_{n}(t)=\lambda S t
$$

until the system reaches its stationary state, where sand loss and sand injection through nucleation are equal. This takes exactly $\tau_{d}$, the time used to define both $\xi$ and $\eta$ (see eq. (10)). After $\tau_{d}$ the number of dunes is stable:

$$
N_{n}(t)=\xi^{*} S
$$

We compare the theoretical evolution of the total number of dunes to the simulation on Fig. 6, where we can see the perfect agreement between the two of them. 


\section{The dense regime}

\subsection{Field characteristics}

a)

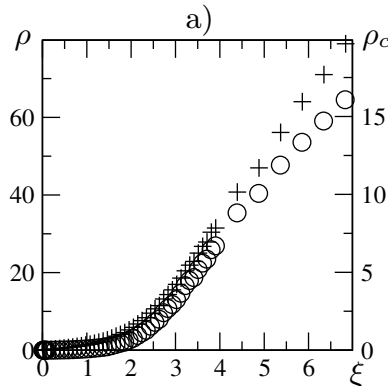

c)

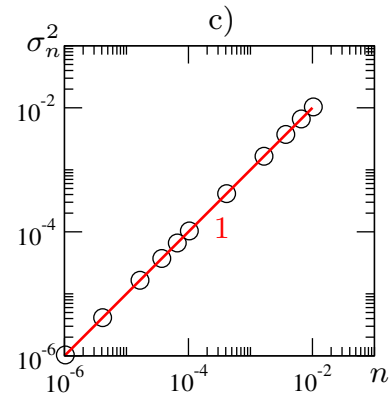

b)

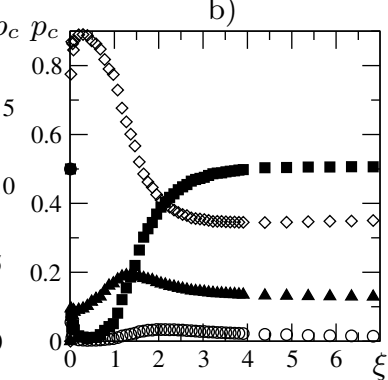

d)

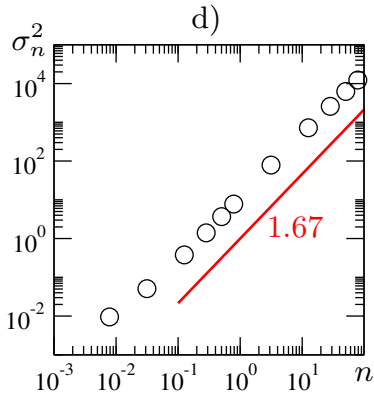

Fig. 7. a) Mean density of the field $(+)$ and mean collision rate by surface unit (o) as functions of $\xi$. b) Proportion of each type of collision, as a function of $\xi$ : (०) Coalescence; ( $\mathbf{\Delta})$ Sand redistribution with $s=1$; $(\diamond)$ Sand redistribution with $s<1$; ( $)$ Fragmentation. c\&d) Variance of the fluctuations of the local number of dunes $n$, as a function of $n$, for $\xi=0.0977$ $(\mathrm{c})$, and $\xi=6.84(\mathrm{~d})$.

For $\xi>1$, the system still reaches a stationary state, but the density of the field explodes (Fig. 7a). The regime is thus called dense [15]. As the density increases, the collision rate increases too (Fig. 7a). Dunes live longer, therefore they are likely to interact with each other and to collide. Among the collisions, fragmentation becomes the most frequent type for dense fields (Fig. 7b), so there is a feedback effect on the density. As the collision rate increases, the fragmentation becomes the most frequent type and collisions generates more dunes in the field, leading to even more collisions.

This increase of the field density is not homogeneous. The field becomes structured, with very dense areas elongated along the wind direction separated by much more dilute inter-regions (Fig. 10a), whereas the dilute field is homogeneous. These dense areas are clusters of dunes, which appear progressively as the field becomes denser. We study the fluctuations of the local density by looking at the relation between $n$, the mean number of dunes in a square of side $d$, and its variance $\sigma_{n}^{2}$, when $d$ changes. The relation between $\sigma_{n}^{2}$ and $n$ is usually a power law $\sigma_{n}^{2} \propto n^{\beta}$. In the dilute field the value of the exponent $\beta$ is one, and the fluctuations follow the normal $\sigma_{n}^{2} \propto n$ law. It confirms that in this regime the field is purely randomly populated (Fig. 7c). On the contrary, the dense field exhibits "giant" fluctuations of the local density, since the exponent $\beta$ is greater than one: we observe a $\sigma_{n}^{2} \propto n^{1+0.67}$ behaviour (Fig. 7d). These fluctuations may be related to what appears on systems where collective phenomena emerge, such as Vicsek models [19] where similar exponents on giant number fluctuations are found.

\subsection{Global scales}

\subsubsection{Fluctuations}
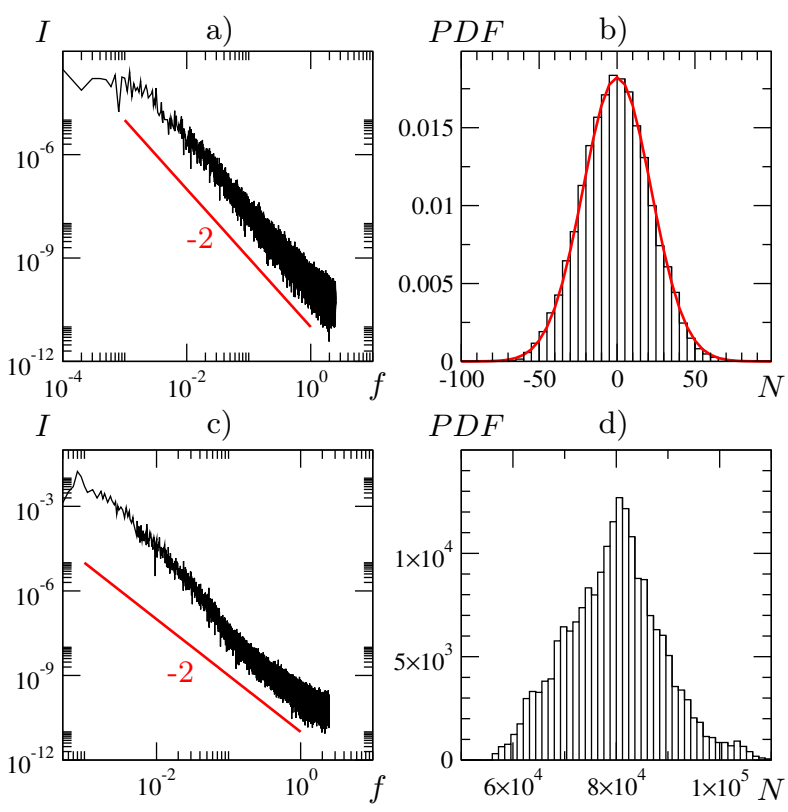

$P D F$

d)

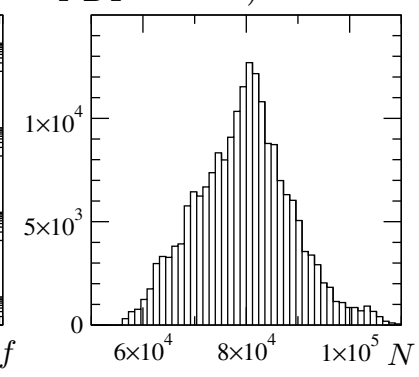

Fig. 8. a) Spectrum and b) distribution of the fluctuations of the number of dunes in the system, for $\xi=0.488\left(5 \times 10^{5}\right.$ events). The red line shows a gaussian fit of the distribution. c) Spectrum and d) distribution of the fluctuations of the number of dunes in the system, for $\xi=2.93\left(2.5 \times 10^{6}\right.$ events $)$.

Global quantities of the field fluctuate around their mean value. In the dilute regime, these fluctuations exhibit a usual brownian $I \sim f^{-2}$ spectrum (Fig. 8a) with a gaussian distribution (Fig. 8b). It means that the dynamics of this regime is essentially noise-driven, i.e. driven by the random nucleation of dunes. This is not surprising, since no other dynamics than the rapid fading of dunes takes place in the system, because of the short lifetime of the dunes.

In the dense field, the spectrum of the fluctuations is no longer characteristic of a brownian noise, but exhibits an over-representation of small frequencies (Fig. 8c), i.e. long time scales. On the same time, the distribution of the 
fluctuations (Fig. 8d) is no longer gaussian and presents large deviations. Long time scales and large deviations are easily linked to the dynamics of the clusters, which drags the system away from its stationary state by giant bursts of the number of dunes, and slowly relaxes because of sand loss.

\subsubsection{Case of the total volume of sand $Q$}

The interacting dynamics between dunes has strong effects on the small scale phenomenology. But these features can also be traced at the field's scale.

The total volume of sand in the system, defined as:

$$
Q=\sum_{k=1}^{N} w_{k}^{3}=N\left\langle w^{3}\right\rangle
$$

is not conserved at the scale of the field because of the dune nucleation and the sand loss, due to the effect of both the loss rate $\Phi$ and the minimum size $w_{m}$. This quantity is thus strongly related to the out of equilibrium dynamics of the system, and its behaviour signs very precisely its change. Indeed, the difference between dilute and dense dynamics in the system is obvious on the evolution of $Q$ (Fig. 9a). As $Q$ depends on the volume of dunes, it will be mechanically affected by any change on the large dunes population, whereas it will require massive modifications of the small dunes population to change its behaviour. In the dilute regime, the size distribution does not change much but for small dunes only (few collisions, leading to a weak erosion of the large dunes population and few little dunes). Thus $\left\langle w^{3}\right\rangle$ is roughly constant. $Q$ is therefore proportional to the stationary number of dunes $N$ in the field, and thus increases linearly with $\xi$ ( $c f$. Part 4$)$, as shown on Fig. 9b. The theoretical slope, given by the mean volume of the dilute regime $\bar{V}=5 \times 10^{-4}$, fits very well the simulation data. $Q$ keeps this linear behaviour much longer than any other quantity of the field, as it is only sensitive to strong modifications of the size distribution.

It is only when $\xi>1$ that the population is largely transformed and $Q$ is affected. What happens then is an odd feature of the model: the total volume of sand saturates, and then slowly decreases. If we allow ourselves to a simple analogy, the system reacts like a spring with two different regimes. In the dilute field, the system selects a steady state with more and more sand as $\xi$ increases, just like a spring in its linear mode spreads more and more as heavier weights are suspended to. But this behaviour does not go to infinity: at some point, the system begins to resist to the external forcing. $Q$ saturates and slowly decreases (Fig. 9a). For the spring, it is as if beyond the linear mode of low deformations there was a non-linear effect, that makes its stiffness increase with the spreading, to the point it actually makes the spring shorten. For the model, this feature signs the highly non-linear aspect of the system dynamics.

As for the previous discussions, this change of behaviour is only a consequence of the fragmentation process. The
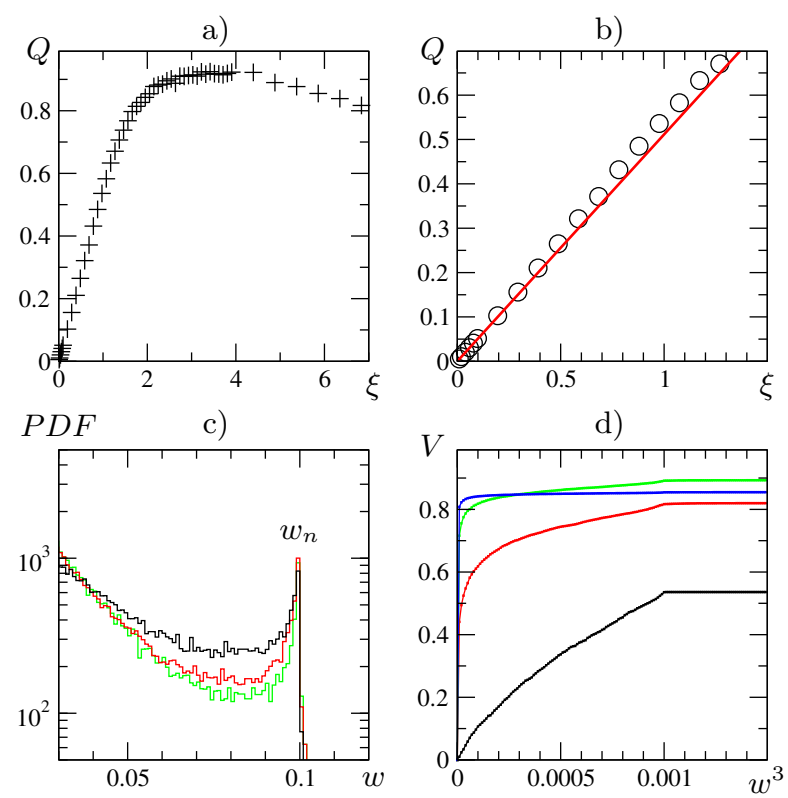

Fig. 9. a) Total volume of sand $Q$ as a function of $\xi$. b) Linear behaviour of $Q$ for small $\xi$. Comparison between the simulations (o) and the theoretical strict dilute regime (-). c) Erosion of the size distribution in the dense regime, as $\xi$ increases : $2.93(-), 5.37(-), 6.84(-)$. d) Cumulative distributive function of the volumes of dunes, for $\xi=0.977(-), 1.95(-), 2.93$ $(-), 6.84(-)$. This shows how each population contributes to the total volume $Q$.

large dunes which are responsible for the linear behaviour of $Q$ are no longer able to maintain their isolated dynamics. Apparition of the collisions leads to the emergence of a peak of small dunes (see Part. 7), and drastic increase of the global sand loss. Indeed, as the number of dunes explodes these new dunes are much closer to the minimum size, therefore likely to disappear. Moreover, in the interval between the peak of small dunes and the nucleation mode lies a consistent density of dunes of intermediate sizes. This population arises directly because of the cascade of collisions from the nucleation size $w_{n}$ to the peak size $\tilde{w}$. They are the intermediate state between the two typical sizes, and can be rather large dunes. Therefore any change of their distribution will affect $Q$. Indeed, as the field gets very dense, the collision dynamics becomes more and more efficient and erodes this population (Fig. 9c). This increases again the sand loss, making its effect exceed the forcing, and thus tends to make $Q$ saturate and then decrease. The large dunes population eventually shrinks, and becomes insignificant. It contributes less and less to the total volume of sand, as seen on Fig. 9d, and eventually the peak population at $\tilde{w}$ remains the only significant one. 


\section{Spatial structuring in the dense regime}

Spatial structuring in clusters is one of the two main features that appear in the dense regime (Fig. 10a), and is responsible for its peculiar dynamics. Its development as $\xi$ - and therefore the density - increases can be tracked by its effect on the spatial correlation of the density. We define a density correlation $a$ for both directions:

$$
\begin{aligned}
& a_{x}(d)=\frac{\langle\rho(x) \rho(x+d)\rangle-\langle\rho(x)\rangle^{2}}{\left\langle\rho(x)^{2}\right\rangle-\langle\rho(x)\rangle^{2}} \\
& a_{y}(d)=\frac{\langle\rho(y) \rho(y+d)\rangle-\langle\rho(y)\rangle^{2}}{\left\langle\rho(y)^{2}\right\rangle-\langle\rho(y)\rangle^{2}}
\end{aligned}
$$

Along the $x$ direction the correlation decreases very fast and becomes zero at a length scale $\sim w_{n}$, confirming that pretty much nothing happens in the transverse direction. However, along the wind direction $y$ the correlation is not negligible (Fig. 10c). Its decreasing with the distance $d$ is not purely exponential, it is therefore not possible to simply extract an accurate correlation length through a fit. However we can study its evolution by determining for each $\xi$ the distance $d_{c}$ at which the correlation reaches a certain value $a_{0}$ (Fig. 10d). For $\xi<1$, the correlation length is too small and falls under the resolution of the algorithm. For $\xi>1, d_{c}$ increases and saturates for high values of $\xi$. This seems to indicate that the structuring of the field reaches a maximum.

In order to study the dynamics of these structures, we define a cluster formally as a population of dunes which interact with each other, and thus form a connected tree of interactions, disconnected from the rest of the dunes. As clusters are very dense, sand capture is very important inside such structures. This interactions sustain small dunes by providing sand, and so they keep the cluster cohesive. Dunes on the upwind front are the only one which actually lose sand. Dunes in the front grow due to the sand incoming from the ones behind them, then slow down and get caught up, and so on. They are also responsible for the sharp edges of the clusters. Dunes may be cast aside by collisions, but if they do they lose the sand supply and thus quickly vanish. We test a model without sand capture. In this model, clusters do not appear (Fig. 10b). The quick decrease of the correlation of the density along the wind direction confirms the lack of spatial structuring in such a system (Fig. 10c).

Clusters are the response of the system to balance sand loss and sand gain. As there is no global sand influx, each barchan - if isolated - can only disappear. Thus the "equilibrium" state of the system would be an empty field. In the dilute regime, the sand loss is large enough for dunes to quickly vanish without interacting. As $\xi$ increases, dunes lose less and less sand, their lifetime increases and collision dynamics appears. As we saw, this leads to the fragmentation of the dunes, via the clusters. In these structures sand is more or less conserved, except at the upwind frontier. Through fragmentations, the number of dunes increases and, as each one of them loses a constant volume $\Phi$ per unit of time, the potential total sand loss increases. As
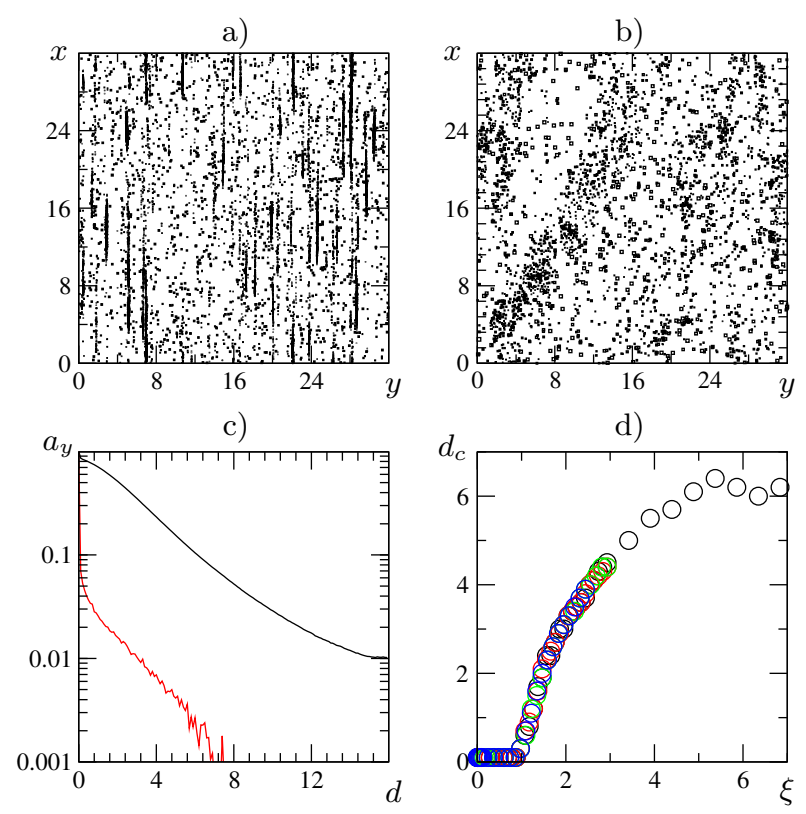

Fig. 10. a) Aspect of a dense field for $\xi=2.93$. Each point is a dune. b) Aspect of the field for a simple model without eolian sand capture between dunes, for $\xi=2.93$. c) Correlation along the wind direction for $\xi=2.93$, for the full model $(-)$, and the simple model without eolian sand capture between dunes (-). d) Correlation length as a function of $\xi$ for $a_{0}=0.1$ and different lengths of the field: $L=32$ (०), 64 (०), 128 (०), 256 (o).

dunes in a cluster exchange sand, there is sand loss only at the upwind side. Because clusters are not strictly aligned along the wind direction, and often make a small angle with it, or have complex shapes, thus sand loss occurs on a frontier of significant length. The accumulation of collisions inside the cluster also dissipates dunes via the effect of the minimum size $w_{m}$. As a result, the apparition of clusters increases the sand loss for the whole system.

In the dense regime, this response gets heavier as $\Phi$ decreases (i.e. $\xi$ increases). Eventually, all dunes in the field become part of a cluster, and therefore interacts with their neighbours, as seen on Fig. 11a. More than an increase of the number of clusters, it is the number of dunes each cluster gathers that increases (Fig. 11b). The clusters grow in size, and one can expect that the longest reaches the dimension of the system. That actually happens for small, finite systems (Fig. 11c). However, these long clusters do not connect with themselves, for two reasons. First, their overall direction is slightly bent, therefore their end do not catch their beginning through the periodic boundaries. Secondly, the big clusters are formed by a very transient reunion of smaller clusters. They usually aggregate and decompose within a few time steps, a too short time to get a self-interacting cluster. Furthermore, when we increase the length of the system, the size of the largest cluster grows but slower than $L$ (Fig. 11d), meaning that 

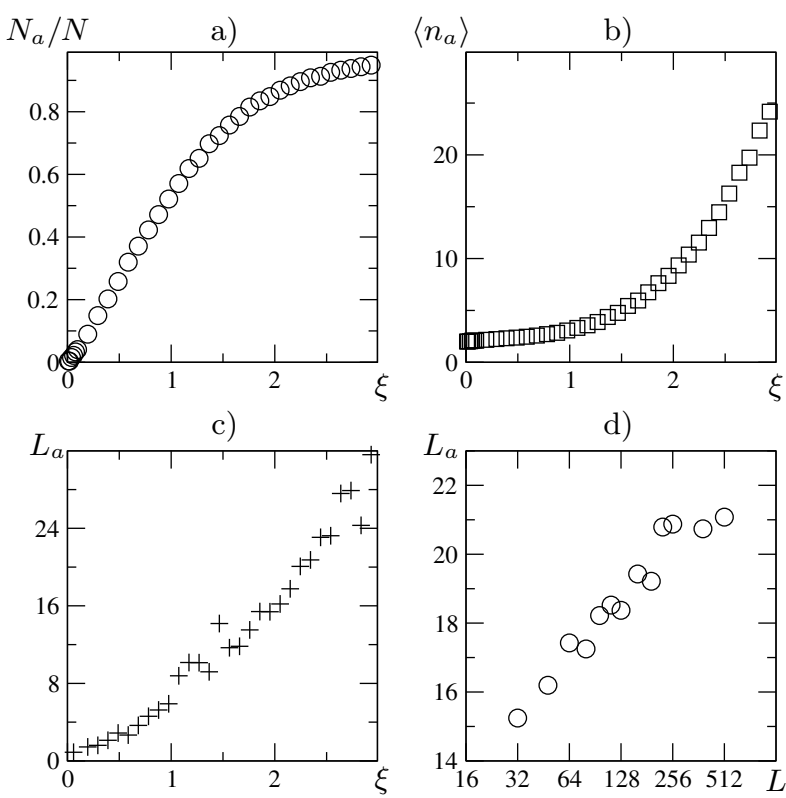

Fig. 11. a) Proportion of the total number of dunes that belongs to a cluster, as a function of $\xi$. b) Mean number of dunes in a cluster as a function of $\xi$. c) Length of the longest cluster in the field, averaged on measures done every $10^{3} \Delta t$, as a function of $\xi$, for $L=l=32$. d) Mean length of the longest cluster in the field, measured every $10^{3} \Delta t$, as a function of the size of the system, for $\xi=1.95$.

for a system of infinite length the possibility of looping clusters vanishes.

\section{Size selection}

\subsection{A collective phenomenon due to collisions}

In addition to spatial structuring, when $\xi$ increases the mean size of the dunes decreases and saturates to a small value, distinct from the minimum size $w_{m}$ (Fig. 12a). The size distribution becomes strongly peaked around a selected width $\tilde{w}$, close to but distinct from the minimum size $w_{m}$ (Fig. 13a). Nevertheless, the distribution still exhibits a mode around the nucleation size $w_{n}$, similar to the nucleation mode of the distribution in the dilute regime.

The cascade from the nucleation mode to the new mode is a consequence of the main mechanism occurring in the dense field. The collision rate in the dense field is very high, and the main collision type is the fragmentation (see Fig. 7). This type of collision increases the number of dunes in the field, but as the total volume of sand is conserved during a collision, it produces smaller dunes. The accumulation of collisions thus tends to reduce the mean size in the field. Dunes nucleated go through lots of collisions, and the products of these collisions gradually go from $w_{n}$ to $\tilde{w}$, which produce the "fat-tail" region inbetween the two modes of the distribution. Furthermore, this effect is not linear. The fragmentation collision produces two small dunes for each large one it splits. Hence, the population of small dunes grows fast, which generates the peak.
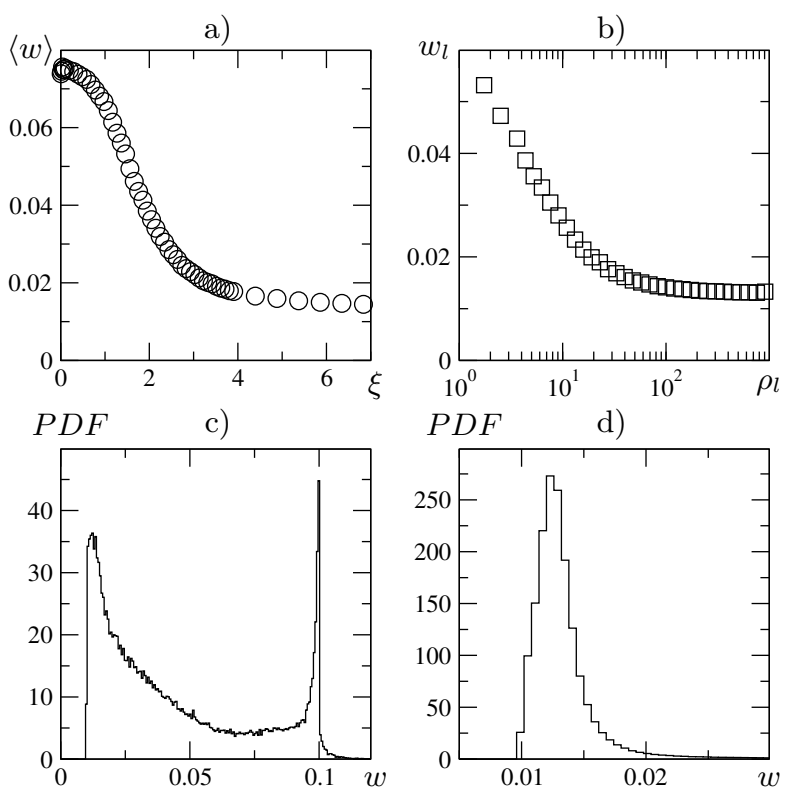

Fig. 12. a) Mean size in the field, as a function of $\xi$. b) Relation between the local mean size and the local density. c) \& d) Size distributions for $\xi=6.84, \eta=1260, \delta=\Delta=0.1$, for clusters with respectively less and more than 5 dunes.

Since clusters are the densest parts of the field, it is pretty clear that this process mainly occurs inside these structures. This assumption is confirmed by plotting the conditional size distributions inside and outside clusters (Fig.12c\&d), and the relation between local mean size and density (Fig. 12b). Small dunes are generated and thus gathered in clusters, where they do not lose sand anymore, and have a long lifetime. As a result, small dunes stay a very long time in the field, whereas large dunes cannot live long before getting hit and split by collisions. Interclusters region then gather only nucleated dunes, small dunes that escape from clusters, and remain of vanishing clusters.

\subsection{Modeling the size selection}

\subsubsection{Random collisions approximation}

We have shown that the accumulation of fragmentations is the main mechanism for the selection of a particular size. Clusters apparition can be seen as a collective phenomenon, in response to the decreasing efficiency of sand loss in each dune. Though the dense field is very heterogeneous, clusters themselves seem to be quite homogeneous. 
Therefore we can imagine that collisions are occurring at random inside clusters. From the collision diagram (Fig. 3) we can compute the theoretical occurrence probability for each type of dune, as a function of the size of the downwind colliding dune. Having the numerical distribution of the sizes, we can calculate these probabilities for the stationary, dense field, and compare them to the numerical values (Table 3 ). Though the agreement is not perfect, the result is good enough to prove that, in the first approximation, the collision process inside clusters is indeed random.

\begin{tabular}{|c|c|c|}
\hline & Analytic & Numerical \\
\hline Coalescence & $0.6 \%$ & $2 \%$ \\
\hline Fragmentation & $53.5 \%$ & $50 \%$ \\
\hline Sand redistribution $s=1$ & $38.9 \%$ & $35 \%$ \\
\hline Sand redistribution $s<1$ & $6.9 \%$ & $13 \%$ \\
\hline
\end{tabular}

Table 3. Comparison between analytic and numerical probabilities for each type of collision.

\subsubsection{Mean-field models}

We push forward this hypothesis by building a mean-field model in which pairs of dune randomly collide. All dunes collide at each time step. The type of the collision is randomly chosen according to the probabilities $p_{c}, p_{f}$ and $p_{r}=1-p_{c}-p_{f}$. The new volumes of the dunes are also randomly chosen, but their choice follows the conservation of the total volume during a collision. The effect of the minimum size $w_{m}$ is kept, and at each time step dunes of size $w_{n}$ are nucleated. The system reaches a stationary state in which the size distribution of the population (Fig. 13b), though not exactly the same as the one from the full model (Fig. 13a), exhibits similar characteristics: a peak for a small size, close but distinct from the minimum size $w_{m}$, a second mode at the nucleation size $w_{n}$, and a cascade between the two of them.

Though simpler than the full model, we did not manage to analytically solve such a mean-field model, because of the highly non linear terms due to collisions phenomenology. In order to get some analytic insights of how random events accumulation can produce a size selection, we reduce again the phenomenology to a simple markovian process for particles. The volume of a dune then evolves according to:

$$
V_{n+1}=c V_{n}
$$

where its value at the time $n+1$ is given by its value at the time $n$ multiplied by a random coefficient $c$ that includes the collisions phenomenology. This process is equivalent to randomly choose a point $(\pi, \theta)$ in the collision diagram (Fig. 3) at each time step, and compute the effect on the
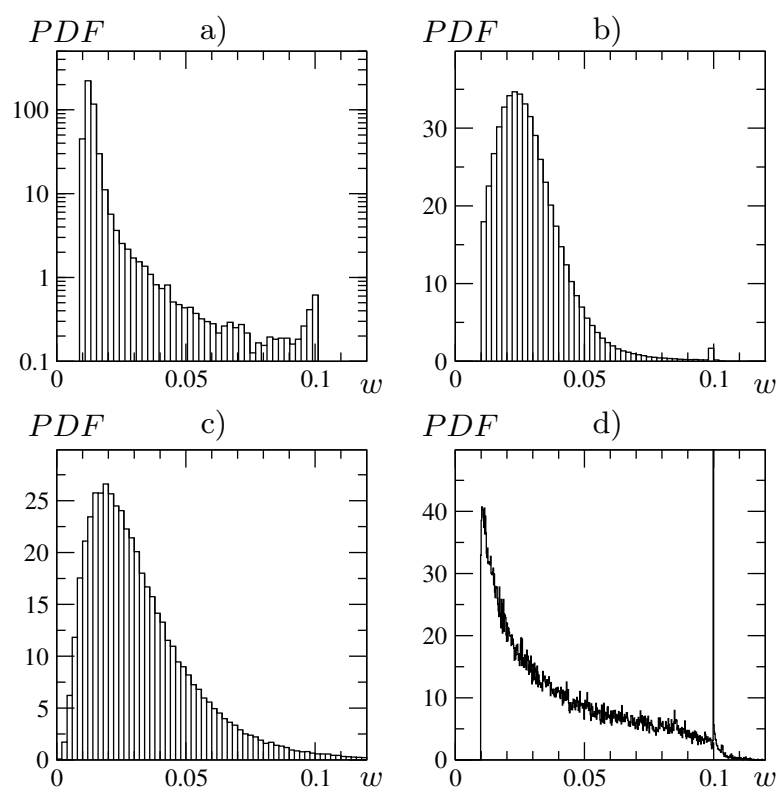

Fig. 13. Comparison of the size distributions: a) Agentbased model, b) Mean-field model with random collisions, for $p_{c}=0.2, p_{f}=0.5, p_{r}=0.3$. c) Markovian model. d) Non conservative markovian model. All distributions are normalised so that their integral is 1 . The vertical scale in a) is logarithmic.

dune. The $c$ operator can therefore be explicitly written:

$$
\begin{aligned}
c(\pi, \theta) & =\left(1+\theta^{3}\right) H_{C}(\pi, \theta) \\
& +\left(1-\frac{1+\theta}{2}+\pi\right) H_{F}(\pi, \theta) \\
& +\left(1-\theta+\theta^{3}\right) H_{T}(\pi, \theta) \\
& +\left(1-\frac{1+\theta}{2}+\pi+\theta^{3}\right) H_{P}(\pi, \theta) \\
& +1 \times H_{I d}(\pi, \theta)
\end{aligned}
$$

The point chosen in the collision diagram is define by its coordinates $(\pi, \theta)$, which are random numbers evenly distributed in the interval $[0,1]$. The $c$ operator contains five terms, namely in order of occurrence in eq. 38: coalescence, fragmentation, sand redistribution with full overlap, sand redistribution with partial overlap, plus the case when the point $(\pi, \theta)$ falls out of the domain of existence for collisions. These terms are calculated from the effect of each collision on the volume of the dune (see eq. 6), and delimited by $H_{K}(\pi, \theta)$ functions, which return 1 when the point $(\pi, \theta)$ is in the region $K$ of the collision diagram, and 0 otherwise. The main advantage of this model is that the volume of a dune at a time step $n$ can be explicitly written:

$$
V_{n}=V_{0} \prod_{k=1}^{n} c_{k}
$$

Numerical computation of this model generates a log-normal distribution for the sizes of the dunes (Fig. 13c). This 
would be consistent with the field observations reported in [5]. The $c_{k}$ are independent, positive, identically distributed, random variables which distribution is square integrable. Although their distribution is not gaussian, it seems that the central limit theorem applies in this case, so that the distribution of their product, and $V_{n}$, tend to a log-normal as $n \rightarrow \infty$. This model serves only as an interpretation for the barchan field behaviour, so we are not interested in mathematically proving this convergence. This model, though it explains very simply how random collisions manage to select a size, is incomplete. The maximum of the distribution tends to zero when the number of collisions tends to infinity, meaning that the selected size tends to zero.

Indeed, the model lacks the vanishing size and the nucleation process. We include these features into the previous model, to build a non conservative markovian model for dunes. In this model, at a time $n$, populations of dunes with different size distributions cohabit in the field. Dunes injected at the time $n$ have gone through only one iteration of the markovian process, and thus their size follow the distribution of $c_{n}$. Dunes that were injected a step before follow the distribution of $c_{n} \times c_{n-1}$, and so on. Distributions do not go down to $p\left(\prod_{k=1}^{n} c_{k}\right)$, as dunes reach the limit value $w_{m}$ after a finite number of iteration $u_{m}$. This imposes an effective cutoff to the progression in $n$. The global size distribution will therefore be the sum of these distribution, stopped at this effective cutoff $u_{m}$ :

$$
p(V)=\sum_{u=1}^{u_{m}} p\left(\prod_{k=1}^{u} c_{k}\right)
$$

This distribution is thus the sum of distributions that converge to log-normals, each taken at successive steps of this convergence. Because of this cutoff, the maximum value of $p(V)$ does not tend to zero, but is blocked to a certain value $\tilde{w}$ close to but distinct from $w_{m}$ (Fig. $13 \mathrm{~d}$ ). The size selection is thus a collective phenomenon, the result of the competition between the effect of the minimum size $w_{m}$ and the sum of perfectly random processes that converge to log-normal distributions.

\section{Conclusion}

We have presented in this paper a precise study of an agent-based model for barchan fields. This geophysical system exhibits interesting features, in particular a spatial structuring and a size selection in its dense regime. Our model takes into account the well known physics of isolated dunes, and two interaction processes: remote sand exchange and collisions. The model is intrinsically non conservative, because of sand loss and dune nucleation. However, it always leads - in the range of parameters we studied - to a stationary state for the field. These stationary states range from dilute to dense as the control parameter $\xi$ - which compares sand loss to dune nucleation - increases. The dilute field is sparse, homogeneous, with dunes that barely interact with each other and which sizes are close to the nucleation size. The dense field is strongly heterogeneous and composed of dunes that interact a lot. A typical size emerges from these interactions. Due to this particular dynamics, the total volume of sand in the field increases with $\xi$ in the dilute regime, then saturates and decreases in the dense regime.

The peculiar features of real barchan fields - spatial structuring and size selection - prove to be related to the interactions between dunes. As the stationary state of the field gets denser, local high densities are unstable and engenders clusters of dunes through avalanches of collisions. Sand recapture is then responsible for the growth and the upkeep of these well-defined, elongated clusters. Their apparition creates then giant fluctuations of density in the field.

Clusters, as they are very dense structures, cause an increase of the collision rate. Among collisions, the fragmentation type is the most frequent. We showed that the accumulation of collisions leads to the selection of a new, small size for the dunes. Collision type frequencies can be accurately calculated by a purely random phenomenology, allowing us to develop a mean-field model based on such a random phenomenology, and a simpler non conservative markovian model. Both show a size selection similar to what occurs in the agent-based model.

Size selection and spatial structuring are not independent phenomena. Collisions generate clusters, and in return clusters are responsible to the accumulation of collisions that enables the size selection. The increase of dunes lifetime inside a cluster enhance this size selection, by allowing small dunes to live a much longer time. These small dunes, escaping from clusters, ensure then the apparition of new clusters.

We can conclude from this study that dune-dune interactions, and in particular fragmenting collisions and sand recapture, are key mechanisms that can explain the global, non trivial behaviour of real fields. We showed that their presence is also sufficient to trigger both the spatial structuring in dense corridors and the size selection by the local density. The paradox between the instability of isolated barchans and large fields observations can be explained by considering the effect of these non trivial, non linear interactions between barchans. Moreover, barchan fields can then be linked to the wide class of systems where large-scale physics emerges through collective behaviour and self-organisation.

Only a fraction of the possible behaviours of the model has been explored. The model has a large phase diagram, controlled by six dimensionless parameters: the limit overlaps $\varepsilon_{t}$ and $\varepsilon_{p}$, the two aspect ratios $\delta$ and $\Delta$ and the two typical times ratios $\xi$ and $\eta$. In this study, only one parameter - the sand loss $\Phi$ - has been modified, meaning that in the $(\xi, \eta)$ space, we only have explored a $\eta \propto \xi$ line. The exploration of the rest of the diagram of such a fully non conservative system should reveal some other odd and interesting features. 


\section{References}

1. R. A. Bagnold. The physics of blown sand and desert dunes. Springer Netherlands, 1971.

2. M. C. Bourke, J. E. Bullard, and O. S. Barnouin-Jha. Aeolian sediment transport pathways and aerodynamics at troughs on Mars. Journal of Geophysical Research-Planets, 109(E7), Jul 132004.

3. J. Radebaugh, R. Lorenz, C. Spencer, and Cassini Radar Team. Terrestrial analogues of longitudinal dunes on Titan. In Planetary Dunes Workshop: A Record of Climate Change, 2008.

4. B. Andreotti, P. Claudin, and S. Douady. Selection of dune shapes and velocities - Part 1: Dynamics of sand, wind and barchans. European Physical Journal B, 28(3):321-339, Aug 2002.

5. O. Durán, V. Schwämmle, P. G. Lind, and H. J. Herrmann. The dune size distribution and scaling relations of barchan dune fields. Granular Matter, 11(1):7-11, Jan 2009.

6. H. Elbelrhiti, B. Andreotti, and P. Claudin. Barchan dune corridors: Field characterization and investigation of control parameters. Journal of Geophysical Research-Earth Surface, 113(F2), Mar 2008.

7. P. Hersen, K. H. Andersen, H. Elbelrhiti, B. Andreotti, P. Claudin, and S. Douady. Corridors of barchan dunes: Stability and size selection. Physical Review E, 69(1, Part 1), Jan 2004

8. H. J. Herrmann. Evolution and shapes of dunes. Comptes Rendus Physique, 3(2):197-206, Mar 2002.

9. K. Kroy, G. Sauermann, and H. J. Herrmann. Minimal model for aeolian sand dunes. Physical Review E, 66(3, Part 1), Sep 2002.

10. O. Durán, E. J. R. Parteli, and H. J. Herrmann. A continuous model for sand dunes: Review, new developments and application to barchan dunes and barchan dune fields. Earth Surface Processes and Landforms, 35(13):1591-1600, Oct 2010.

11. V. Schwämmle and H. J. Herrmann. A model of Barchan dunes including lateral shear stress. European Physical Journal E, 16(1):57-65, Jan 2005.

12. A. Katsuki, H. Nishimori, N. Endo, and K. Taniguchi. Collision dynamics of two barchan dunes simulated using a simple model. Journal of the Physical Society of Japan, 74(2):538-541, Feb 2005

13. D. Zhang, C. Narteau, and O. Rozier. Morphodynamics of barchan and transverse dunes using a cellular automaton model. Journal of Geophysical Research-Earth Surface, 115, Sep 2010.

14. A. R. Lima, G. Sauermann, H. J. Herrmann, and K. Kroy. Modelling a dune field. Physica A-Statistical Mechanics and its Applications, 310(3-4):487-500, Jul 2002.

15. M. Génois, S. Courrech du Pont, P. Hersen, and G. Grégoire. An agent-based model of dune interactions produces the emergence of patterns in deserts. Geophysical Research Letters, 40(15):3909-3914, 2013.

16. P. Hersen, S. Douady, and B. Andreotti. Relevant length scale of barchan dunes. Physical Review Letters, 89(26), Dec 232002.

17. P. Hersen. Flow effects on the morphology and dynamics of aeolian and subaqueous barchan dunes. Journal of Geophysical Research-Earth Surface, 110(F4), Nov 162005.

18. H. Elbelrhiti, P. Claudin, and B. Andreotti. Field evidence for surface-wave-induced instability of sand dunes. Nature, 437(7059):720-723, Septembre 2005
19. H. Chaté, F. Ginelli, G. Grégoire, F. Peruani, and F. Raynaud. Modeling collective motion: variations on the Vicsek model. European Physical Journal B, 64(3-4):451-456, Aug 2008. 